Portland State University

PDXScholar

\title{
Calibrating an Intracranial Pressure Dynamics Model With Clinical Data - A Progress Report
}

Wayne W. Wakeland

Portland State University, wakeland@pdx.edu

James McNames

Portland State University

Brahm Goldstein

Oregon Health \& Science University

Follow this and additional works at: https://pdxscholar.library.pdx.edu/sysc_fac

Part of the Biomedical Commons

Let us know how access to this document benefits you.

\section{Citation Details}

Wakeland, W., Goldstein, B., and McNames, J. Calibrating an Intracranial Pressure Dynamics Model with Annotated Clinical Data--a Progress Report. Presented at the 26th Annual International Conference of the IEEE Engineering in Medicine and Biology Society, 2004 in San Francisco, California.

This Post-Print is brought to you for free and open access. It has been accepted for inclusion in Systems Science Faculty Publications and Presentations by an authorized administrator of PDXScholar. Please contact us if we can make this document more accessible: pdxscholar@pdx.edu. 


\title{
CALIBRATING AN INTRACRANIAL PRESSURE DYNAMICS MODEL WITH ANNOTATED CLINICAL DATA--A PROGRESS REPORT
}

\author{
W. Wakeland ${ }^{1}$, J. McNames ${ }^{2}$, B. Goldstein ${ }^{3}$ \\ ${ }^{1}$ Systems Science Ph.D. Program, Portland State University, Portland, Oregon, USA \\ ${ }^{2}$ Biomedical Signal Processing Laboratory, Electrical and Computer Engineering, Portland State University, Portland, \\ Oregon, USA \\ ${ }^{3}$ Complex Systems Laboratory, Division of Pediatric Critical Care, Oregon Health \& Science University, Portland, Oregon, \\ USA
}

\begin{abstract}
We describe the calibration of a computer model of intracranial pressure (ICP) dynamics to correspond with annotated clinical data taken from a patient being treated for elevated ICP due to a traumatic brain injury. The research protocol employed during treatment includes adjusting the elevation of the head of the bed, adjusting the ventilator settings to induce mild hyperventilation and hypoventilation, and adjusting the height of the cerebrospinal fluid drainage system. The model behavior corresponds to the experimental data quite well in the case of the changing the head of the bed, but less well in the case of changing the ventilator settings.
\end{abstract}

Keywords-intracranial pressure (ICP), traumatic brain injury (TBI), dynamic modeling, annotated clinical data

\section{INTRODUCTION}

Traumatic brain injury (TBI) is the leading cause of death and disability in children, causing more than $50 \%$ of all childhood deaths. Each year, more than 150,000 pediatric brain injuries result in about 7,000 deaths and 29,000 children with new, permanent disabilities. Costs attributed to treating pediatric TBI in the USA exceed $\$ 12$ billion dollars annually. A recently published evidencebased medicine review reports that almost all therapies and drugs used to treat children with life-threatening brain injury are based upon weak if any pathophysiologic evidence and have not been proven to be safe or effective [1]. And, despite a plethora of treatment options for reducing elevated intracranial pressure (ICP), poor outcomes still result in many cases, often due to secondary brain injury. The complex processes at work during TBI are only partially understood. Questions have been raised, for example, even about the common practice of elevating the patient's head to lower their ICP [2].

Over the past thirty years, a variety of computer models for calculating ICP have been published in the literature [3-6]. Our contribution to this collection was presented at EMBS 2003 [7]. These models use differential equations to calculate the pressure at different points within the system, taking into account the volumes and compliances of the various vessels and compartments.

Although useful mathematical results and insights have been reported for these models, their impact on clinical practice has been modest, due in part, perhaps, to their complexity. Researchers have attempted to address

This work was supported in part by the Thrasher Research Fund. this by simplifying their models as much as possible (cf. [8]). Others have focused on making fluid volumes and flows more explicit, and have incorporated explicit parameters and logic that is relevant to clinical research and practice [7].

One of the primary challenges facing the researcher when applying his or her model to the clinical environment is proper interpretation of the clinical data. Retrospective data often lacks the accurately time-synchronized annotations that would be needed to allow the data to be used to estimate parameters and refine model logic. This problem is rectified in the present research by using a laptop-based portable data acquisition system (PDAS) that captures the signal and parametric data from the patient monitoring equipment, and also has software to allow the researcher to enter time synchronized annotations about initiation of therapies and other events of interest. PDAS is an adaptation of our previously reported clinical data acquisition system [9].

The methodology section of the paper describes the research protocol for collecting annotated clinical data, and discusses how the base ICP dynamic model was modified to represent therapeutic interventions contained in the research protocol. The results section of the paper provides several sample segments of clinically annotated data, discusses the model calibration process, and shows the resulting model behavior.

\section{METHODOLOGY}

\section{A. Experimental Protocol for Data Acquisition}

For TBI patients with consent, three mild physiologic challenges are given: 1) altering the head of the bed (HOB), 2) altering the respiratory rate (VR), and 3) altering the height of the external cerebrospinal fluid (CSF) drainage system (when present).

The degree of HOB elevation is routinely maintained at $30^{\circ}$. The protocol adjusts the degree of HOB elevation in random order between $0^{\circ}, 10^{\circ}, 20^{\circ}, 30^{\circ}$ and $40^{\circ}$; with each value being held for 10 minutes. ICP and other physiological signals (electrocardiogram, blood pressure, etc.) are recorded continuously during these procedures.

Baseline $\mathrm{PaCO}_{2}$ is routinely kept between 35-38 $\mathrm{mmHg}$. This is monitored using a combination of arterial blood gas sampling and end-tidal $\mathrm{CO}_{2} \quad\left(\mathrm{ETCO}_{2}\right)$ monitoring. The challenge protocol adjusts $\mathrm{PaCO}_{2}$ by increasing (hyperventilation) and then decreasing (hypoventilation) the minute ventilation, in random order, 
keeping the tidal volume fixed and changing the respiratory rate to achieve an $\mathrm{ETCO}_{2}$ of $[-3$ to $-4 \mathrm{mmHg}]$ and [ +3 to $+4 \mathrm{mmHg}$ from baseline level. At each target $\mathrm{ETCO}_{2}, \mathrm{PaCO}_{2}$ is checked via arterial blood gas to ensure that the $\mathrm{PaCO}_{2}$ has changed by 3-4 mmHg. If not, the respiratory rate is further adjusted, and $\mathrm{ETCO}_{2}$ and $\mathrm{PaCO}_{2}$ are rechecked to ensure that the target $\mathrm{PaCO}_{2}$ value has been achieved. Each target level is maintained for 15 minutes.

Routine CSF drainage via ventriculostomy catheter is done on a continuous basis with an external CSF drainage bag placed at the patient's eye level. For these patients, the height of the external CSF drainage system is varied in random order from $-8,-4,0,+4$, to $+8 \mathrm{~cm}$ above eye level. The amount and rate of CSF drainage is measured every $15 \sec \mathrm{x} 4$, and then every minute. Each level is maintained for 10 minutes.

\section{B. Modifications to the Base Model}

The model described in detail in [8] was modified to reflect the research protocol. Eq. 1 indicates the rate of CSF drainage. The pressure differential due to changing the HOB is shown by eq. 2 .
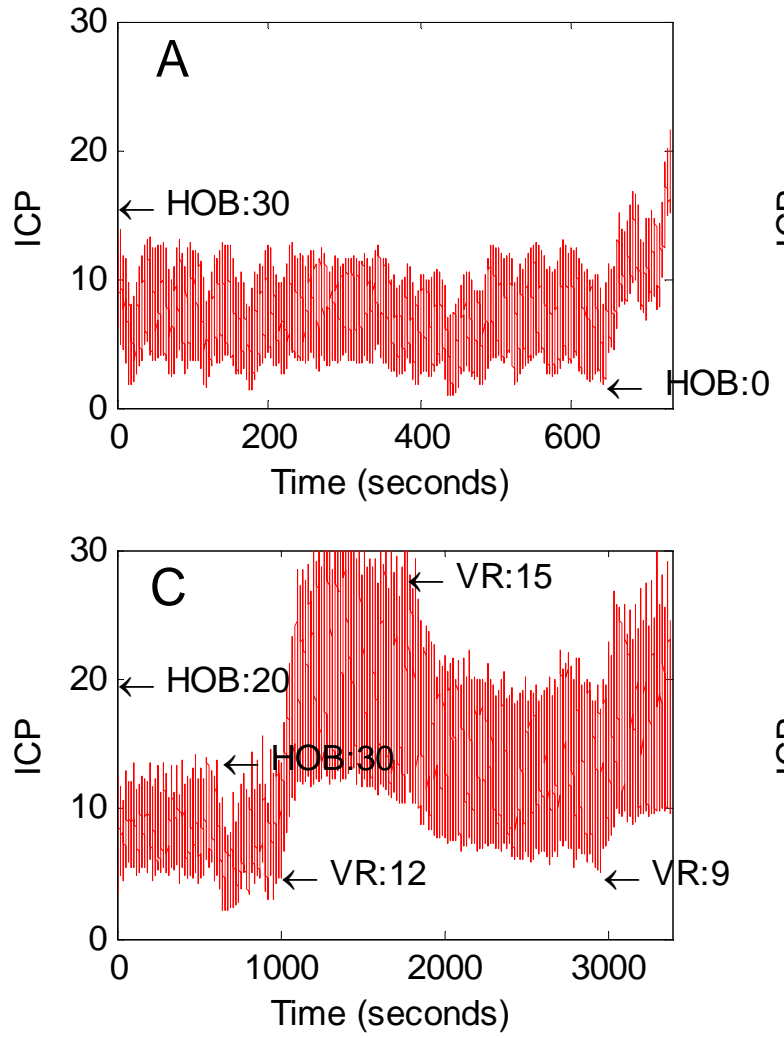

$R_{C S F}=\frac{\left(P_{v}-P_{t}\right)}{r_{c}}$

where: $R_{C S F}=$ CSF drainage rate, $P_{v}=$ ventricular pressure (ICP), $P_{t}=$ pressure at drain terminus ( $=\mathrm{fn}$. of its elevation), and $r_{c}=$ resistance of the catheter.

$\Delta P=d * \sin (\theta) * \frac{m m H g}{m m H_{2} O}$

where: $\Delta P=$ the pressure difference, $d=$ the distance between the heart and the head, and $\theta$ is the HOB angle.

$\Delta P$ is subtracted from both the systemic arterial pressure and the systemic venous pressure to determine the external pressures at the cranium.

The impact of changing the ventilator rate is much more complex (cf. [10]). We model this very simplistically, as indicated in Eqs. 3 and 4.

$$
\begin{aligned}
& f_{i}=k_{1}+\alpha *\left(P_{a} C_{2}-S\right) \\
& P_{a} C O_{2}=\operatorname{MOV}\left[\left(k_{2}-\beta * V R\right), t_{c}\right]
\end{aligned}
$$

Where: $f_{i}=$ indicated blood flow, $\mathrm{k}_{1}=$ baseline blood flow, $\alpha=$ flow multiplier, $\mathrm{PaCO}=$ actual $\mathrm{CO}_{2}$ press., $S=$ setpoint for $\mathrm{CO}_{2}$ press., $k_{2}$ and $\beta$ are est. conv. factors, $V R$ is the respiration rate in breaths per minute, and $t_{c}$ is the time constant for the moving avg. (MOV).
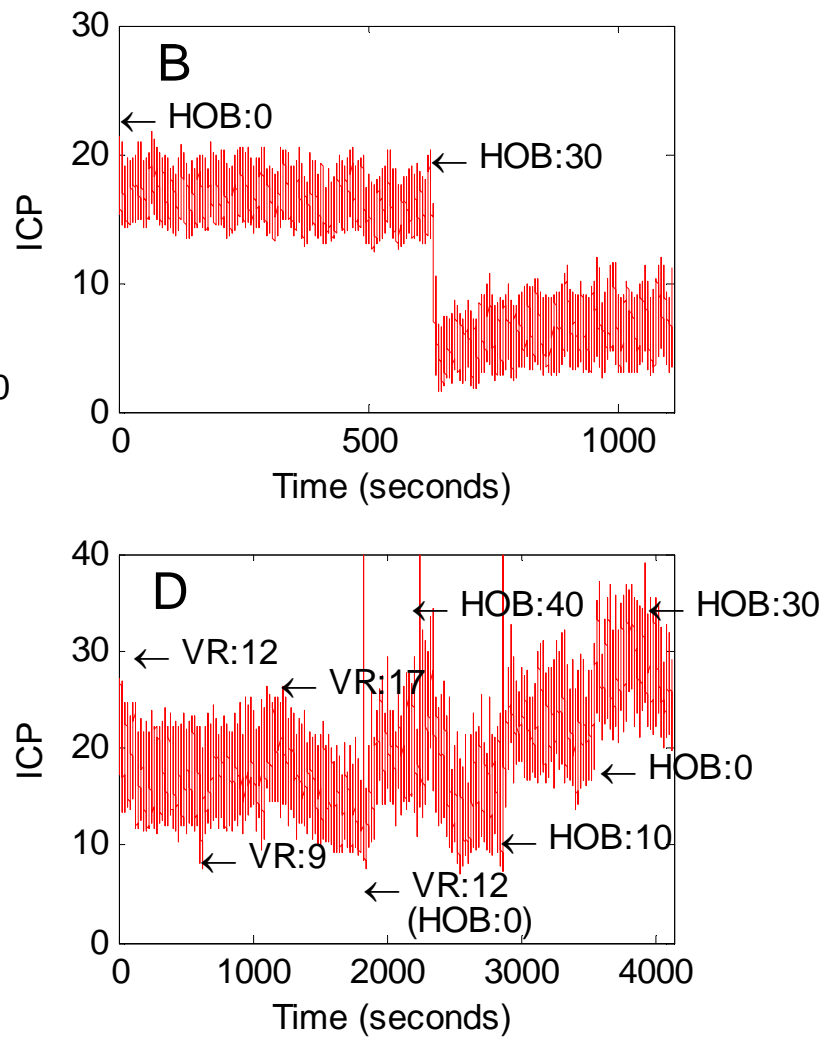

Figure 1: Annotated clinical data. Each plot shows the ICP (intracranial pressure) signal, sampled at $125 \mathrm{~Hz}$. Annotations are added from the log file as follows. The tip of the arrowhead indicates the time of the annotation. HOB is head of the bed angle in degrees. VR is the respiration rate in breaths per minute (bpm). Plot A shows the response to HOB being lowered from 30 to 0 degrees. Plot B shows the response to an HOB increase from 0 to 30 degrees. Plot $\mathrm{C}$ shows HOB being changed from 20 to 30 degrees, followed by changes in VR from baseline (15 bpm) to $12 \mathrm{bpm}$; back to $15 \mathrm{bpm}$; then to $9 \mathrm{bpm}$. Plot D, recorded a day later, shows a sequence of VR changes according to the experimental protocol, followed by a sequence of HOB changes according to the experimental protocol. 
1: Modeled ICP

2: Actual ICP EpisodeA

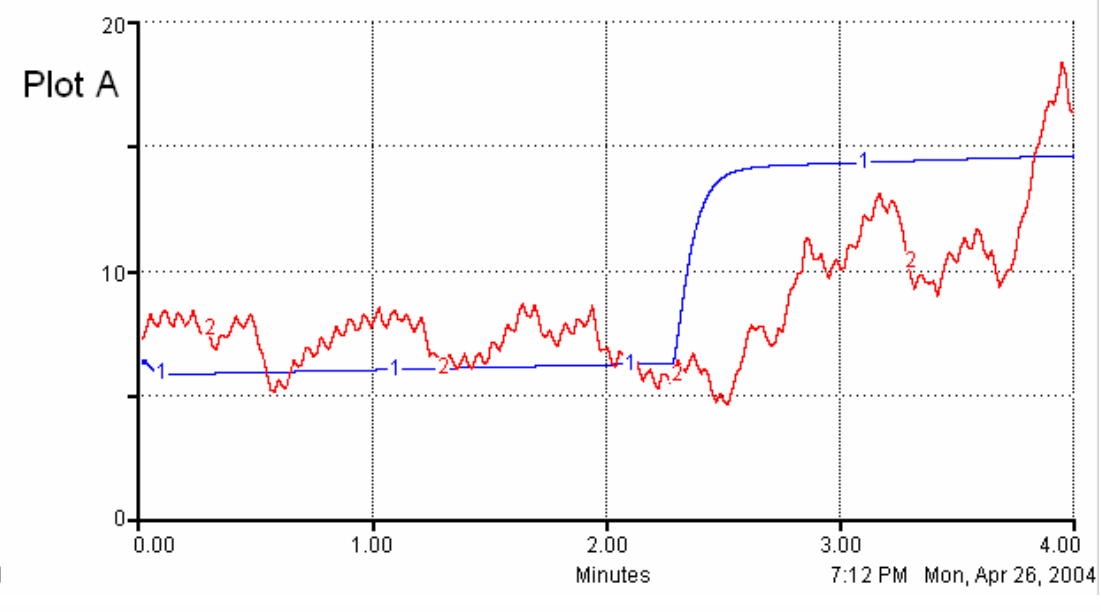

1: Modeled ICP

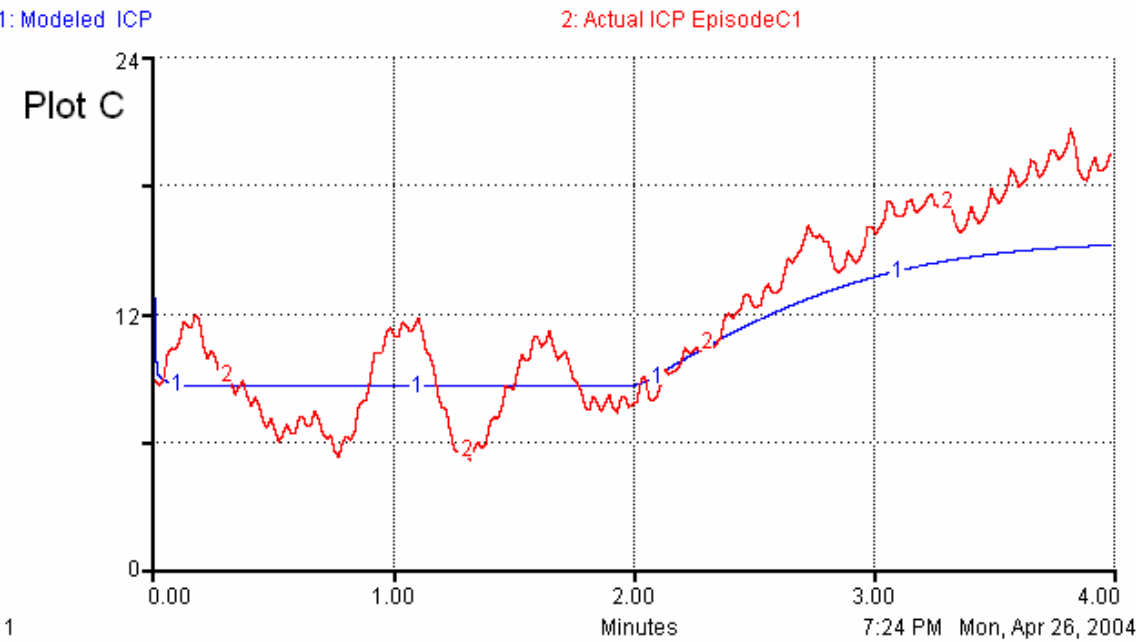

1: Modeled ICP

2: Actual ICP Episode日

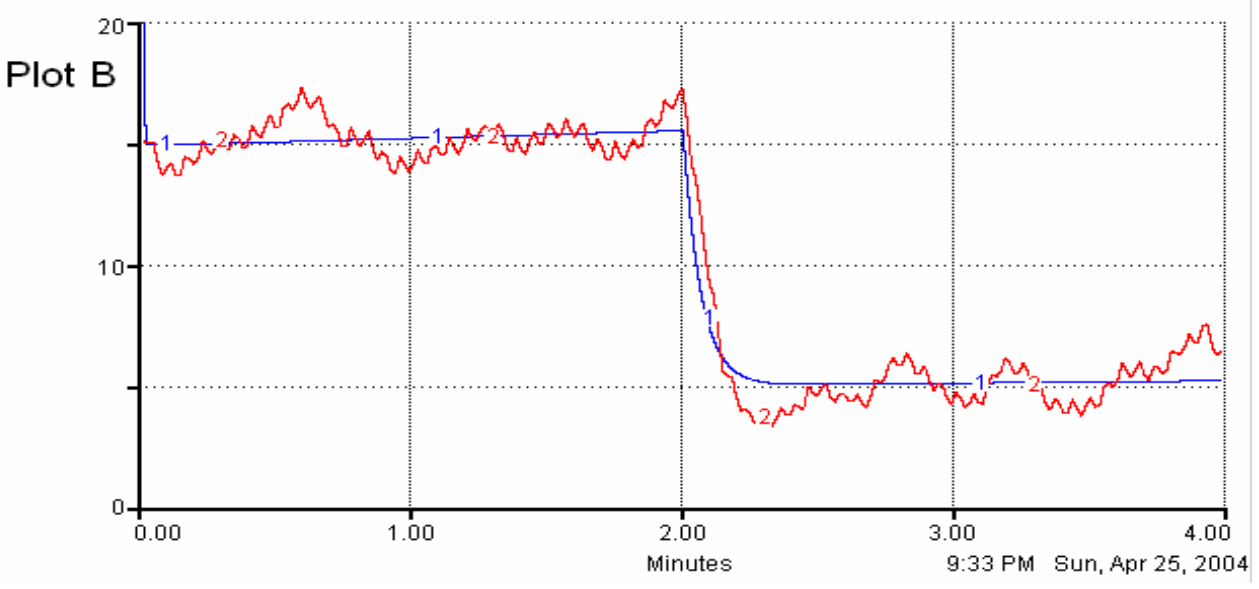

1: Modeled ICP

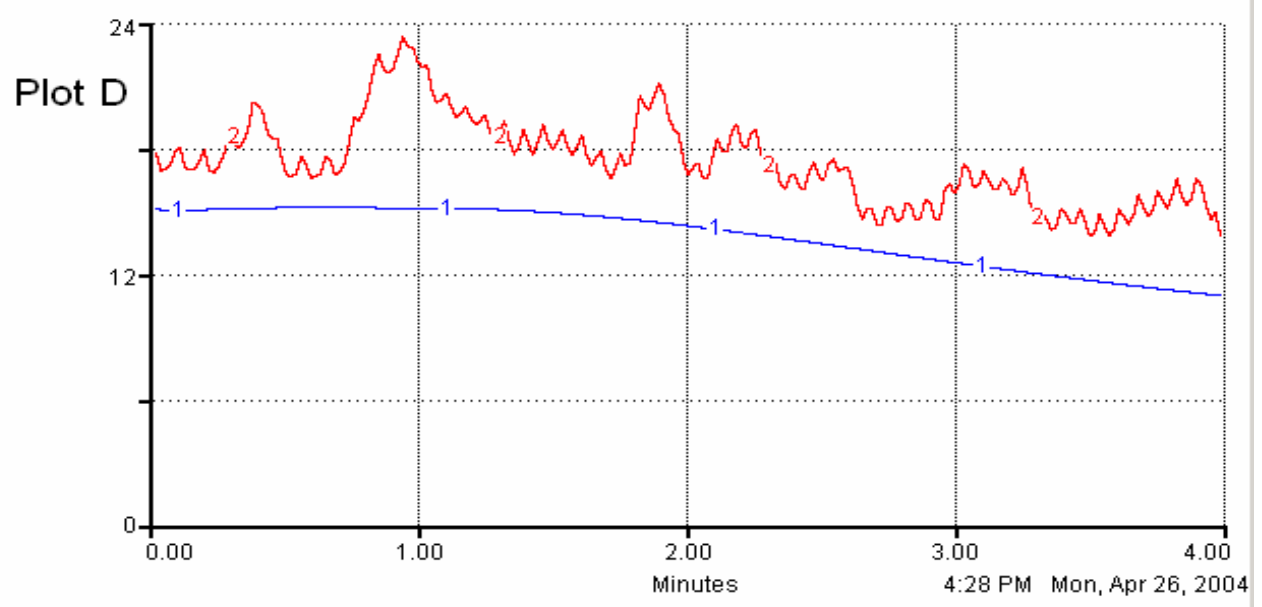

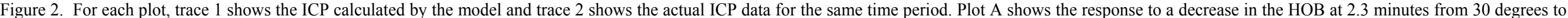

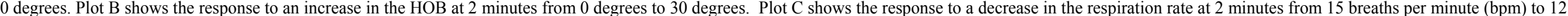
$\mathrm{bpm}$. Plot $\mathrm{D}$ shows the response to increasing the respiration rate at 2 minutes from $12 \mathrm{bpm}$ to $15 \mathrm{bpm}$. 
Actual flow and indicated flow are compared by the autoregulation logic in the model that adjusts smooth muscle tension in order to constrict or dilate the blood vessels to adjust the flow.

\section{RESULTS}

\section{A. Clinically Annotated Data}

Figure 1 shows four periods of clinically recorded ICP data.

Annotations indicate when the HOB and VR were changed. Figure $1 \mathrm{~A}$ indicates a period of relative stability in ICP, followed by a rapid increase due to the HOB being lowered from $30^{\circ}$ to $0^{\circ}$. Figure $1 \mathrm{~B}$ shows the ICP response when the $\mathrm{HOB}$ is maintained at 0 for ten minutes and then increased back to $30^{\circ}$. The reduction in ICP is rapid and profound. Figure $1 \mathrm{C}$ begins with the $\mathrm{HOB}$ at $20^{\circ}$ and ICP at approximately $9 \mathrm{mmHg}$. The annotations indicate that the patient was being hyperventilated during this period. The HOB is raised to $30^{\circ}$, causing a modest reduction in ICP. VR is then reduced to 12 breaths per minute (bpm). ICP rises rapidly. The target $\mathrm{PaCO}_{2}$ was quickly achieved, and after 10 minutes, VR was increased to $15 \mathrm{bpm}$. ICP falls, but not as rapidly. This is due in part to the ventilator automatically adjusting the tidal volume to compensate. The researcher made additional adjustments to achieve the target $\mathrm{PaCO}_{2}$. VR was then reduced to $9 \mathrm{bpm}$ and ICP begins to increase. Figure 1D shows a sequence of VR adjustments followed by a sequence of HOB adjustments. This fourth sequence of tests took place one day later.

\section{B. Calibration of the Base Model to Clinical Data}

The model was calibrated to approximate the clinical behavior exhibited during several of the episodes shown in Figure 1. To begin with, a modest subdural hematoma of $6 \mathrm{~mL}$, increasing at $.5 \mathrm{~mL}$ per minute, was consistent with the data. A moderate degree of brain swelling could also explain the observed data. In addition, a modest increase in CSF absorption resistance was indicated. At 2.3 minutes, $\mathrm{HOB}$ is lowered. The model indicates a rapid increase in ICP. Parameter $d$ in Eq. 2 was estimated to be $40 \mathrm{~cm}$. Actual ICP increases more slowly, and the response lags the stimulus by about .5 minute. Figure $2 \mathrm{~A}$ shows the model behavior superimposed with the corresponding clinical reference data.

Figure 2B shows the response to raising $\mathrm{HOB}$ to $30^{\circ}$. The response to this change is rapid, both in the model and in the patient. To obtain this excellent agreement between the model and reality, the size of the hematoma in the model was allowed to continue to increase at .5 $\mathrm{mL}$ per minute, and $d$ was revised to $45 \mathrm{~cm}$.

Figure $2 \mathrm{C}$ indicates the response to a decrease in $\mathrm{VR}$ at 2 minutes. $\alpha$ in Eq. 3 is estimated to be $75 \mathrm{ml} / \mathrm{mmHg}$, and $S=34 \mathrm{mmHg}$. For Eq. $4, k_{2}=64 \mathrm{mmHg}, \beta=2$ mmHg-breaths/minute, and $t_{c}=2.5$ minutes. The model does not reflect the full magnitude of the patient's response to this stimulus, due in part to the simplistic implementation of this reflex in the model. We utilize a single auto regulatory mechanism, whereas in fact there are several mechanisms, each of which has a different characteristic time constant [10]. Figure 2D shows how ICP changes in the model in response to increasing VR. Actual clinical data is also shown for reference.

We have made important progress, and the results are encouraging, but many of the mechanisms in the model need further refinement. Further, although the System Dynamics modeling platform utilized during model development was an excellent environment for model conceptualization, its support for data analysis and parameter estimation is very limited. The model has been ported to Simulink, and future work will be done using that superior computational platform. We are also linking the model to nonlinear control algorithms so that model parameters can be estimated in a more automated fashion.

\section{REFERENCES}

[1] P. Adelson, et al. Guidelines for the acute medical management of severe traumatic brain injury in infants, children and adolescents. Ped Crit Care Med 2003. 4 [3].

[2] M. Rosner. Pathophysiology and management of increased intracranial pressure. In: Neurosurgical Intensive Care, B.T. Andrew (ed), McGraw-Hill, Inc, 1993: 57-112.

[3] A. Marmarou, K. Shulman. Computer modeling of CSF Pressure/ Volume and its relationship to hydrocephalus. Intracranial Pressure. 1972. M. Brock, H. Dietz. Berlin. Springer-Verlag: 275-279.

[4] M. Czonyka, S. Piechnik, H. Richards, P. Kirkpatrick, P Smielewski, J. Pickard. Contribution of mathematical modeling to the interpretation of bedside tests of cerebrovascular autoregulation. $J$ Neurosurg 1997;63:721-731.

[5] M. Ursino, M. Iezzi, N Stocchetti. Intracanial Pressure Dynamics in Patients with Acute Brain Damage: A Critical Analysis with the Aid of a Mathematical Model. IEEE Transactions on Biomedical Engineering 1995;42:529-540.

[6] M. Ursino, E. Magosso. Role of tissue hypoxia in cerbrovascular regulation: a mathematical modeling study. Ann. of Biomedical Engr. 2001. 29:563-574.

[7] W. Wakeland, J. McNames, M. Aboy, D. Holleman, B. Goldstein. Modeling intracranial fluid flows and volumes during traumatic brain injury to better understand pressure dynamics. Annual Int'l Conf. of IEEE Engr. in Medicine and Biology - Proc. Cancun, Mexico, pp 402405, 17-21 September 2003.

[8] M. Ursino, C.A. Lodi. A Simple mathematical model of the interaction between intracranial pressure and cerebral hemodynamics. J.. Appl. Physiol.,1997. 82[4]: 1256-1259.

[9] Goldstein et al. A physiologic data acquisition system and database for the study of disease dynamics in the intensive care unit. Crit Care Med 2003. 31: 433-441.

[10] J.W. Phillis (ed.) The Regulation of Cerebral Blood Flow. CRC Press. 1993. Boca Raton. 